\section{G231(P) THE MEDICAL ASSISTANTSHIP SCHEME: IMPROVING PATIENT CARE AND TRAINING FOR MEDICAL STUDENTS}

A Sharma. Paediatric Emergency Department, Royal Wolverhampton NHS Trust, Wolverhampton, UK

\subsection{6/archdischild-2020-rcpch. 198}

Introduction Paediatric Emergency Departments (ED) continue to face increasing pressure to deliver high quality care to patients. Ensuring units are adequately staffed with the correct skill mix is a constant challenge. Newly qualified doctors face the prospect of working and developing in this environment, putting into practice what they learnt at medical school whilst trying to acquire resilience.

This pilot project was designed to help reduce pressures in the paediatric ED and enable students to learn skills by employing them as 'medical assistants' (MA), better preparing them for life as a new doctor.

Methods In collaboration with the local medical school, six MAs were appointed in July 2018 to the emergency department to work alongside a paediatric ED consultant between $6 \mathrm{pm}$ and $11 \mathrm{pm}$ weekdays and $3 \mathrm{pm}$ to $11 \mathrm{pm}$ at weekends. They performed a range of clinical, clerical and nursing tasks. Paediatric ED consultants and MAs completed daily surveys as well as a final end-of- pilot survey. Nursing staff and patients were asked to complete a one-off survey. In addition, data looking at attendances, time to see clinician, time in the department and breeches were analysed.

Results From October 2018 to May 2019 there was a statistically significant increase in paediatric ED attendances compared to the same period the year before. Despite this increase, the key metrics for flow in the paediatric ED (i.e. triage times, times to see a clinician and time in the department) did not change significantly.

From a qualitative perspective, the consultants felt that the presence of the MA reduced their stress levels and enabled them to provide a better level of care. Nursing staff found them useful to nursing and medical staff. Parents commented being seen by well-mannered staff in an efficient manner. The MAs found the experience invaluable towards their personal and professional development.

Conclusion This study demonstrates that the MA scheme can be mutually beneficial to paediatric ED and medical students by providing improved patient care as well as preparing medical students for life as new doctors. We believe this scheme could be easily adapted to other clinical settings in the wider NHS.

\section{G232(P) TRANSCUTANEOUS BILIRUBINOMETER AND HOME PHOTOTHERAPY}

S Mulvaney. Accident and Emergency, Alder Hey Children's NHS Trust, Liverpool, UK

\subsection{6/archdischild-2020-rcpch. 199}

Aims To optimise the investigation of neonatal jaundice within the first two weeks of life in the emergency department (ED), through the guideline led use of a transcutaneous bilirubinometer (TCB). To establish and support a pathway led home phototherapy service in the community setting.

Methods 1 year retrospective case note reviews of:

- all jaundiced babies and all bilirubin samples sent to the lab, from infants between 1 and 14 days, from the ED in 2016.
- use of the transcutaneous bilirubinometer (TCB) in 2017

- babies with uncomplicated jaundice admitted for phototherapy in 2016 and 2017.

2 month review of home phototherapy service established end August 2019, including parent feedback.

Results

- In 2016, 433 separate patient visits were identified, 254/433 (59\%) patients had a bilirubin less than, or equal to 250 .

- Of this 254, the average age was 6 days and average bilirubin 187. 11/254 (4\%) patients had to have bloods repeated due to failure of initial bloods. Average wait time until bloods results was 1 hour and 35 minutes and the average length of stay in the department was $4 \mathrm{hr} 17$ minutes

- 1 year review of established TCB guideline showed a reduction in blood tests by $40 \%$. Wait time from first being seen by a clinician was reduced to 30 min compared to $1 \mathrm{hr}$ $57 \mathrm{~min} .70 \%$ guidance adherence was noted.

- Around 100 babies per year are admitted for an average of 1-2 days with uncomplicated jaundice for phototherapy only.

4 babies have been successfully managed and discharged after 2 days on home phototherapy with excellent feedback.

Conclusions Use of a TCB is NICE evidence based and noninvasive. It optimises patient journey and reduces harm in a cost effective manner.

Currently well babies presenting to the ED with jaundice and requiring phototherapy are admitted for an average of 12 days. The setup of the home phototherapy service allows babies with uncomplicated jaundice to be treated at home with daily visits and blood bilirubin monitoring.

This frees up potential bed spaces, by reducing unnecessary admissions in an infectious environment, whilst minimising parental anxiety.

\section{G233(P) ELECTRONIC PATIENT INFORMATION LEAFLETS: THE FUTURE?}

D Murrell. Paediatric Emergency Department, Addenbrookes Hospital, Cambridge, UK

\subsection{6/archdischild-2020-rcpch.200}

Aim To improve the number of paediatric patients who receive written discharge advice following discharge from the paediatric emergency department in line with clinical standards as set out in Facing the Future: Standards for children in emergency care settings

Method Patient information is generally regarded as improving patient care as it provides relevant health care information, empowers patients in their healthcare decisions and encourages engagement with healthcare services.

An audit of clinical records showed that less than 10\% of patients discharged from the paediatric emergency department received documented written and verbal advice.

Following engagement with clinical staff, steering groups, hospital management and patient information services to investigate the barriers a $\mathrm{QR}$ poster was developed.

The poster enables care givers or patients to get electronic versions of patient information leaflets on a mobile device, broadening both information and media available to patients. 
As a simple design the poster is easy reproducible and using web based analysis tools, monitoring the use is simple, providing useful data on patient engagement, and what information is being accessed. Clinical notes were re-audited over a 6 month period to observe sustained improvement.

Results Since implementation there has been an overall increase of patients receiving documented written and verbal discharge advice to $85 \%$, with an average of 11 clicks of electronic discharge information leaflets a day (as of 19th October 2019).

There was a $22 \%$ reduction in unplanned attendances to the paediatric emergency department over the same time period.

There has been multiple positive feedback, including via social media from both clinicians and patients about the ease of use and quality of information provided.

Other benefits include a rapid way of providing information with a potential reduction in physical leaflets needed, with resource and cost savings to services.

Conclusions The QR poster provides an innovate method of providing relevant and accessible patient information leaflets to patients with patient service experience and potential resource reduction benefits.

\section{G234(P) THE SAFE ADMINISTRATION AND AVAILABILITY OF INTRAMUSCULAR ADRENALINE FOR PAEDIATRIC ANAPHYLAXIS - QUALITY IMPROVEMENT THROUGH SIMULATION}

N Richens, B Miguras. TR, Trainee Research Association for Paediatric Emergency Medicine, UK

\subsection{6/archdischild-2020-rcpch.201}

Introduction Intramuscular adrenaline is a time-critical emergency treatment in the acute management of paediatric anaphylaxis. A low fidelity simulation project was carried out to identify the relative safety of existing emergency kit adrenaline vials compared to pre-filled adrenaline autoinjectors in the management of a simulated anaphylaxis in a 5 year old male.

Method A low fidelity simulation consisting of a paediatric registrar and a paediatric staff nurse familiar with their departments were carried out under timed conditions. 12 Paired trials were carried out across two centres in paediatric emergency departments, paediatric admission units, and paediatric inpatient wards. Each paired trial occurred during the same shift with the same staff. The time from the medical request for a defined dose of $0.15 \mathrm{ml}$ of $1: 1000$ adrenaline to be provided at the bedside in a syringe with an IM needle attached was recorded. Errors in the initially provided dose were immediately identified and the time required for the provision of the correct dose measured.

Results The mean time to completion of the simulation for the emergency kit arm was 138.3 seconds (range 96 - 223 seconds) compared to 33.6 seconds (range 17 - 54 seconds) for the adrenaline autoinjector arm. Incorrect doses of adrenaline were provided to the clinician in 2 of 6 emergency kit simulations. There were no dosing errors in the autoinjector arm. In all cases the nursing staff reported preferring the autoinjector method.

Conclusion This study provides clear evidence for the improved safety of adrenaline autoinjectors over emergency kits in the hospital management of paediatric anaphylaxis. The increased time for the emergency kit arm may partly reflect the availability of various components (vials, syringes, filter and IM needles) in different clinical settings. The significant risk of errors as a consequence of human factors in emergency drug preparation is already well-known and reflected in this study. Following these results, paediatric adrenaline autoinjectors have been adopted universally across both sites. The cost consideration of autoinjectors over adrenaline vials, particularly variation in expiry rate, is important when considering departmental policy change. However, the safety concerns were considered to outweigh the increased cost in these centres.

\section{G235(P) HIGH FREQUENCY OSCILLATORY VENTILATION IN PEDIATRIC PRACTICE: A SINGLE CENTER EXPERIENCE}

R Gabr, M Nishil, J Zahraa. PICU, King Fahad Medical City, Riyadh, Saudi Arabia

\subsection{6/archdischild-2020-rcpch.202}

Introduction High frequency oscillatory ventilation (HFOV) is as a rescue mode of ventilation however, it did not show benefit in many cases.

Objectives To identify which patient are best treated with HFOV and which ones will not improve or deteriorate, and the best way of selection and adjustment of HFOV settings in the first 48 hours.

Study Design: Retrospective observational study.

Settings: One tertiary level PICU.

Subjects: 207 patients under 18 years who were admitted to PICU with respiratory failure and switched to (HFOV) in the period between September 2010 till December 2017.

Intervention: None

Results HFOV was used 241 times. 34 cases were excluded and 207 HFOV episodes were analyzed. The median age was 12 (4, 48 IQR) months with 52.7\% males. Etiology of respiratory failure was classified as diffuse alveolar disease (DAD) and small airway disease (SAD). The most common cause of respiratory failure was ARDS, 100 (48\%) cases then pneumonia, $34(16.4 \%)$ cases, two cases of asthma and 8 with bronchiolitis. Majority had an underlying chronic illness (87.4\%). Oxygenation failure was the most common reason for HFOV (77.3\%).

Over the first 48 hours there was a significant improvement in oxygenation but faster in ventilation and acid base status. Variables that were associated with 48 hours mortality were shorter duration of $\mathrm{CMV}$, lower $\mathrm{pH}$, not using $\mathrm{NMB}$ agents, sepsis, high vasopressor index and hypotension just before switching to or within the 48 hours of HFOV. 48 hours mortality was significantly higher in patients with hematological malignancies (OR 8.73 (1.58-48.06), $p=0.013$ and in the poorly identified group that did not fit into DAD or SAD categories when compared with ARDS (OR 2.73 (1.13$6.58), p=0.030)$. Conventional ventilation settings were not statistically significant between surviving vs non-surviving groups $(p>0.05)$.

Conclusion HFOV has a great value in improving oxygenation and ventilation when conventional mode fails. The underlying disease seems to have a significant impact on the outcome and immune function and malignancies are the main categories. Hemodynamic instability and sepsis are major contributors to worse short-term outcome. 